

\title{
Highly sensitive pseudo-differential ac-nanocalorimeter for the study of the glass transition
}

\author{
Mohcine Laarraj, Rahma Adhiri, Said Ouaskit, Mohamed Moussetad, \\ Christophe Guttin, Jacques Richard, Jean-Luc Garden
}

\section{To cite this version:}

Mohcine Laarraj, Rahma Adhiri, Said Ouaskit, Mohamed Moussetad, Christophe Guttin, et al.. Highly sensitive pseudo-differential ac-nanocalorimeter for the study of the glass transition. Review of Scientific Instruments, 2015, 86 (11), pp.115110. 10.1063/1.4935491 . hal-01257649

\section{HAL Id: hal-01257649 \\ https://hal.science/hal-01257649}

Submitted on 18 Jan 2016

HAL is a multi-disciplinary open access archive for the deposit and dissemination of scientific research documents, whether they are published or not. The documents may come from teaching and research institutions in France or abroad, or from public or private research centers.
L'archive ouverte pluridisciplinaire HAL, est destinée au dépôt et à la diffusion de documents scientifiques de niveau recherche, publiés ou non, émanant des établissements d'enseignement et de recherche français ou étrangers, des laboratoires publics ou privés. 


\title{
Highly sensitive pseudo-differential ac-nanocalorimeter for the study of the glass transition
}

\author{
Mohcine Laarraj,,${ }^{1,2,3}$ Rahma Adhiri, ${ }^{3}$ Said Ouaskit, ${ }^{4}$ Mohamed Moussetad, ${ }^{3}$ \\ Christophe Guttin, ${ }^{1,2}$ Jacques Richard, ${ }^{1,2}$ and Jean-Luc Garden ${ }^{1,2}$ \\ ${ }^{1}$ Institut NÉEL, CNRS, 25 avenue des Martyrs, F-38042 Grenoble France \\ ${ }^{2}$ Univ. Grenoble Alpes, Inst NEEL, F-38042 Grenoble France \\ ${ }^{3}$ Laboratoire d'Ingénierie et des Matériaux (LIMAT), \\ Université Hassan II de Casablanca, Faculté des Sciences Ben M'Sik, Casablanca Maroc \\ ${ }^{4}$ Laboratoire de Physique de la Matière Condensée (LPMC), \\ Université Hassan II de Casablanca, Faculté des Sciences Ben M'Sik, Casablanca Maroc
}

(Dated: September 16, 2015)

\begin{abstract}
We present a nanocalorimeter designed for the measurement of the dynamic heat capacity of thin films. The microfabricated sensor, the thermal conditioning of the sensor as well as the highly stable and low noise electronic chain allow measurements of the real and imaginary parts of the complex specific heat with a resolution $\Delta C / C$ of about $10^{-5}$. The performances of this quasi-differential nanocalorimeter were tested on a model of polymeric glass-former, the PVAc. The high stability and low noise of the device are essential for accurate studies on non-equilibrium slow relaxing systems such as glasses.
\end{abstract}

PACS numbers: Thermal instruments and apparatus, 07.20.-n, Calorimeters, 07.20.Fw, Specific phase transitions, 64.70.-p, Glasses, 64.70.kj, Thermal properties of amorphous solids and glasses: heat capacity, thermal expansion, etc., 65.60.+a, Glasses and polymers, 66.70.Hk,

\section{INTRODUCTION}

A vitreous system could be viewed as a liquid having the properties of a solid. The molecular structure of an amorphous material is that of a liquid while its dynamic is totally different. The mean time scale over which this liquid flows is so high that its macroscopic properties are those of a solid. This notion of timescales involved in glasses and a fortiori during the passage from the liquid to the glass (glass transition) is of fundamental importance to really understand these systems. The glass transition can be studied by means of different analytical methods, among which calorimetry is employed for a long time. Calorimetry gives insights on the thermodynamics involved during the glass transition process. As an example, the heat capacity can be measured during vitrification (passage from the supercooled liquid to the glass) or structural recovery (passage from the glass to the liquid). Moreover, heat released (or absorbed) during the physical aging of a glassy system can be directly recorded by calorimetry. Calorimetry allows one to investigate the phenomenology of the glass transition, and often it has to be complemented by methods mostly probing the phenomenon at a molecular level (e.g. spectroscopic ones). Nevertheless, to understand the thermodynamics involved during the glass transition at a macroscopic scale is of high importance for industry where the control of thermodynamic parameters during formation of new glasses is crucial. Among calorimetric methods used for the study of the glass transition, Differential Scanning Calorimetry (DSC) is certainly the most employed method. The heat capacity of a sample is recorded as a function of temperature while the temperature is scanned at a constant rate (cooling or heating). Moreover, the change of temperature rates in DSC is used in order to probe the dynamic of the process (mean relaxation time of the molecular rearrangements) and to obtain information on the underlying thermal activation process. For about twenty years, new calorimetric methods have been developed where heat is alternatively supplied and released in the sample at a given frequency. As a consequence, the temperature of the sample oscillates at the same frequency. Not only the amplitude but also the phase of the temperature oscillation can be recorded. Owing to these two parameters, it is possible to separate the recorded thermal events in a real and an imaginary parts $[1,2]$. In these oscillating calorimetric methods, the recorded heat capacity can be seen as a generalized dynamic susceptibility like in other spectroscopic methods (dielectric, magnetic, mechanical,... ). The measured heat capacity is not a real quantity as usual, but rather a complex quantity with real and imaginary components obeying the Kramers-Kronig relations. Precursors in the study of the glass transition using these techniques are Birge, Nagel and Christensen [3-5]. With the so-called three-omega method, they showed that the complex heat capacity behaves like complex dielectric susceptibilities when glasses are studied. This dynamic thermal method has been frequently used for investigations of the glass transition $[6,7]$. The second method is the Temperature Modulated Differential Scanning Calorimetry (TMDSC) which results on the addition of a temperature oscillation on the classical scanning rates of DSC $[10,11]$. Since this time until today it has been used a lot for the thermodynamic study of glasses [12-21]. The last oscillating 
method, named ac-calorimetry is less employed for glass studies because it is mostly adapted for small systems generally in the field of low temperature physics. It is however with the fast speed DSC certainly one of the most sensitive for the detection of fine thermal events [22-27]. It has been rarely used for the study of the glass transition [28-31]. In this paper, we present a new pseudo-differential ac-nanocalorimeter dedicated to the thermodynamic and dynamic calorimetric study of the glass transition. This nanocalorimeter combines high resolution with rather broad frequency range. The thermal frequency range which can be used can be comprised between $8 \mathrm{mHz}$ and $3 \mathrm{~Hz}$. The resolution in the measured heat capacity $\Delta C_{p} / C_{p}$ is of the order of $10 \mathrm{ppm}$. The high stability of the electronic chain as well as the temperature regulation stages of the nanocalorimeter allows a high repeatability of the measurement. An appropriate thermal model of the device, which is described in detail here, was developed in order to obtain the real and imaginary parts of the complex specific heat of the sample. Resolution, stability and frequency range allow the obtaining of reliable data on fine thermodynamic and dynamic thermal events on a model of polymeric glass former, the PVAc.

\section{DESCRIPTION OF THE NANOCALORIMETER}

\section{Microfabricated sensor}

The heart of the nanocalorimeter is constituted by a home-made nanocalorimetric sensor. The nanocalorimetric sensor, or measuring cell, is made of two half-cells such as depicted in Fig. 1. One half-cell contains a heater and the other one a thermometer. The sample is compressed between the two half-cells ensuring thermal contacts between heater, sample and thermometer. Each half-cell is composed of a thin $(25 \mu \mathrm{m}$ thick $)$ polyimide membrane for thermal insulation of the sensitive area. These membranes are glued on a copper piece that defines the thermal bath for the measurement. On one half-cell (membrane/copper piece), a thin film heater in copper-nickel is deposited by magnetron-assisted sputtering (thickness of about $500 \mathrm{~nm}$ ). A photolithographic step following by chemical etching in ferric chloride is performed in order to obtain a meander with a measurable resistance of about $250 \Omega$ (see Fig. 1). The copper-nickel film has a temperature coefficient of about few $10^{-4} K^{-1}$ ensuring that the supplied power does not vary a lot on the working temperature range. However, four wires connect the heater resistance such as seen in Fig. 1 in order that during an experiment the voltage and current across the heater are permanently known. The thermal power supplied in the sample along a temperature scan is thus exactly known. On the other half-cell, a thin film thermometer in platinum is deposited by magnetron-assisted sputtering (thickness of about $100 \mathrm{~nm}$ ). A first $10 \mathrm{~nm}$ thick adher-

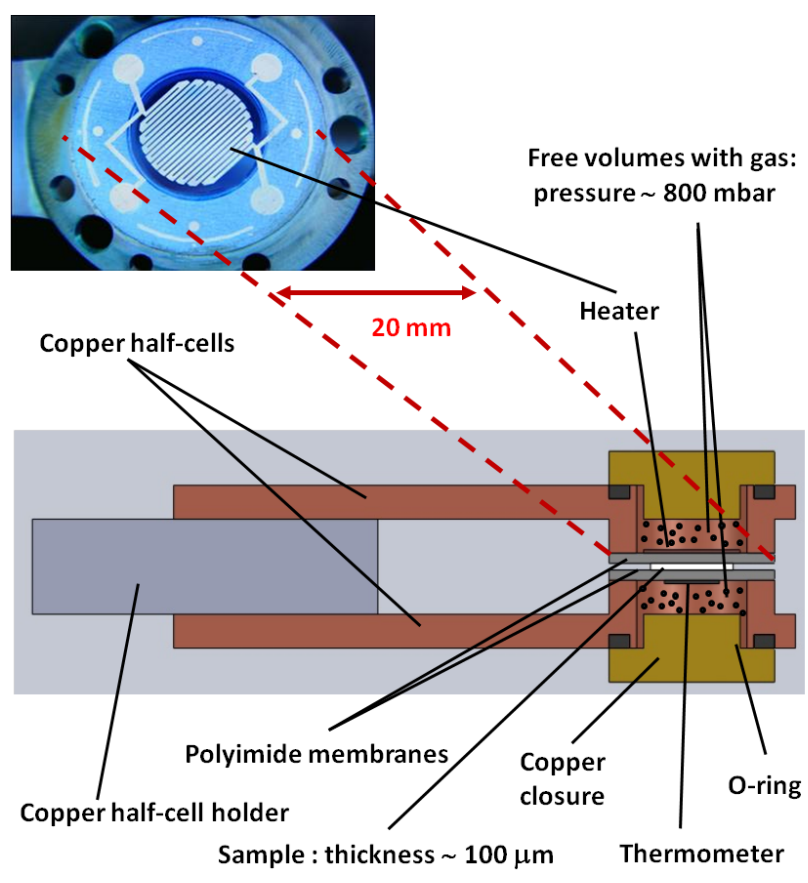

FIG. 1: Sectional view of the two half-cells fixed on the copper half-cell holder. The photography is the top view of the half-cell containing the heater in copper-nickel microphotolithographied on the polyimide membrane.

ence layer of tungsten/titanium alloy is deposited on the polyimide membrane. A photolithographic step followed by dry ionic etching is performed in order to structure a meander with wire of $30 \mu \mathrm{m}$ large and few $\mathrm{cm}$ long zigzagging (the distance between arms of the wire is of $50 \mu \mathrm{m}$ ) on a circular surface of about $0.5 \mathrm{~cm}^{2}$ (diameter of $8 \mathrm{~mm}$ ). The resulting measurable resistance is of about $500 \Omega$. The thickness of the sample can be comprised between few $\mu m$ up to few hundreds of $\mu m$. Its diameter is of $8 \mathrm{~mm}$ (see next sections where the PVAc sample conditionning is depicted). The disk is firstly placed between two thin gold disks (10 $\mu \mathrm{m}$ thick) of the same diameter. This sandwich is then placed on the surface covered by the thermometer (surface of about $0.5 \mathrm{~cm}^{2}$ ) and compressed between the two membranes. These two gold disks ensure homogeneity of the temperature inside the sample/addenda assembly on all the surface of 0.5 $\mathrm{cm}^{2}$. The total measured heat capacity (sample plus addenda) is composed of the two polymer membranes, the heater, the thermometer, the two gold disks and the sample taken over this surface of $0.5 \mathrm{~cm}^{2}$. The absolute value of the heat capacity of such an assembly is on the order of $17 \mathrm{~mJ} / \mathrm{K}$ at room temperature (see table $1)$. Since the value of the thermal conductance generated in the thickness of the two thin membranes between the sample/addenda and the heat bath is small (on the order of $0.14 \mathrm{~mW} / \mathrm{K}$; see table 1 ), then in order to define an appropriate value for the heat exchange coefficient 


\begin{tabular}{|c|c|c|}
\hline \hline$\sharp$ & $C_{p}(m J / K)$ & $K(m W / K)$ \\
\hline Gold & 2.2 & NA \\
\hline Copper-Nickel & 0.1 & NA \\
\hline Platinum & 0.05 & NA \\
\hline Kapton & 4.16 & 0.14 \\
\hline PVAc & 10.4 & NA \\
\hline Air & NA & 1.2 \\
\hline \hline
\end{tabular}

TABLE I: ( $\sharp$ indicates the type of materials). $C_{p}(m J / K)$ is the heat capacity of the different parts of the microsensor, $K(m W / K)$ is the thermal conductance defined across some different parts of the microsensor. These values are estimated at room temperature.

for ac-calorimetry, two copper closures enclose hermetically a free volume back to each membrane. These free volumes are filled with gas (See Fig. 1). Between the sample/addenda at the center of the membrane and the copper closure, heat propagates across this volume of gas by conduction (radiation is negligeable). The distance of about $1 \mathrm{~mm}$ between the membrane and the copper closure defines a value for the thermal conductance between $1 \mathrm{~mW} / K$ up to three $m W / K$. As a consequence, the thermal relaxation time, $\tau=C / K_{b}$ has a value comprised between 5 to $14 \mathrm{~s}$. These values define the lower limits of the operating frequency range of temperature oscillation respecting quasi-adiabaticity conditions (see Fig. 6). O-rings shown in Fig. 1 ensure hermetic separations between the volumes of gas and the exterior of the cell under vacuum. The gas in each closed volume is at a pressure comprised between 700 mbar and 800 mbar. A gas reservoir of volume of about $10 \mathrm{dm}^{3}$ (volume higher than the two volumes in the cell), placed in the room outside the calorimeter, maintains the pressure at a constant value by means of small pipes and holes in the copper closures (not shown in the figure 1). Under these circumstances, the differential pressure between the back and the front of the two membranes maintains them in good thermal contact with the gold-disks/sample assembly. This reinforces the thermal contact between all the elements of the measured heat capacity ensuring better temperature homogeneity. These thermal contacts and the value of the thermal diffusivity in the membranes and the sample assembly as well as the geometry of the system define an internal relaxation time $\tau_{i n t}$. The value of this internal relaxation time defines for instance an upper limit to the useable temperature oscillation frequency range (see Fig. 6). The copper pieces (half-cell) on which the two membranes are glued and the two copper closures define the temperature reference (thermal reservoir) for the measurement. The four contacts of each metallic films are thus at a given controlled temperature. The possible thermoelectric effects that can affect the mean dc temper-



Gold-plated copper support and thermal link

FIG. 2: Photography of the second microphotolitographied platinum thermometer on a copper holder.

ature measurement are then reduced. A Platinum RTD $10 k \Omega$ sensor of Heraeus company is glued on one of the two half-cells for temperature control and regulation. A heater is positioned in a hole in the copper half-cell holder ensuring scanning temperature rates in the range of 0.1 $\mathrm{K} / \mathrm{min}$ to $10 \mathrm{~K} / \mathrm{min}$. The copper half-cell holder, partially shown in Fig. 1, is in direct thermal contact with the cold source defined by a Peltier thermoelectric module (TEM). With this TEM and the heater/thermometer on the cells and the cell-holder, the temperature of the microfabricated sensor can be controlled in heating or cooling mode in temperature range between $+253 \mathrm{~K}$ to $+423 \mathrm{~K}$. Measurements in quasi-isothermal mode can also been performed. In ac-calorimetry, a dc temperature contribution is measured at the same time that the oscillating temperature contribution. It is in general interesting to electronically remove this dc contribution in order to be able to amplify only the oscillating one. For that purpose, another platinum thin film thermometer, shown in Fig. 2, identical to the one on the membrane, is deposited on a polyimide thin film glued on a copper support. This copper support is in thermal contact with the half-cell holder. As a consequence, during temperature scans, the dc temperatures of the two thermometers follow approximately the same variations. In that case, the use of a Wheatstone bridge for this differential temperature detection, allows the recording of the oscillating temperature contribution from the thermometer on the microsensor only, while the differential dc contribution becomes negligible. This is a pseudo-differential measurement because it concerns only the dc temperature while the ac part is plainly that of the sample/addenda assembly. In the references [24, 30-32] the authors developed true differential ac-nanocalorimeters where at least two thermometers oscillate (one with a sample and the other without) and the differential of the ac-temperature is recorded. The differential mode is interesting in itself because it reduces the dynamics of the electronics (num- 
ber of digits) necessary to record the signal, but in our case since the measurement already occurs at the Johnson noise of the resistive thermometers with few nanovolts per square hertz on millivolts of signal, this does not induce significant gain for the resolution of the measurement. Another point is that this microfabricated sensor, in its current design, is not adapted for a differential mode.

\section{Electronic chain of measurement}

In Fig. 3, the schematic view of the electronic detection chain is represented at the same time than some thermal parts of the nanocalorimeter. The two thin film platinum thermometers are mounted in a Wheatstone bridge. The first one on the nanocalorimetric sensor, called $R_{\Theta 1}$, measures the oscillating temperature contribution of the sample/addenda assembly. The second one, called $R_{\Theta 2}$, is on a copper support connected itself to the half-cell holder (see Fig. 2). It gives the temperature of this support which does not oscillate. The two fixed resistors of the bridge, called $R_{F 1}$ and $R_{F 2}$ are of high quality (Vishay RCK02) with temperature coefficient of only few $\mathrm{ppm} / K$. Since the dc temperature of the sample/addenda assembly inside the sensor is in general different than that of the copper holder of the second thermometer then a resistor (called $R_{F}^{e q}$ ) in parallel to one of the fixed resistor of the bridge is added in order to nullify the output dc component. The value of this resistor can be changed easily in a shielded box located outside the calorimeter. This is done each time that the mean thermal power dissipated in the heater resistor $R_{C H a c}$ has to be changed in order to fix the magnitude of the temperature oscillations at different frequencies. The Wheatstone bridge is polarized with a home-made high quality and high stability $10 \mathrm{~V}$ tension supply based on a Zener VRE310 (Thaler corporation, $6 V p-p(0.1-10 \mathrm{~Hz}), 0.6 \mathrm{ppm} / \mathrm{K})$. After equilibration of the dc component of the bridge, the oscillating voltage is amplified with a low noise high stability dc chopper amplifier ( $A 29$ from EMelectronics company). The input voltage noise of the amplifier is on the order of the Johnson noise of the thermometric resistor (i.e. few $n V / \sqrt{H z} R M S)$. Such as discussed in next sections, the maximum possible frequency is of the order of $3 \mathrm{~Hz}$, and the dynamic of the dc amplifier is high enough to avoid the suppression of a part of the sinusoidal signal even up to this frequency. The amplified signal is then recorded (numerized) with NI-DMM-PXI-4071 cards included in a PXI-Rack from National Instrument company. Labview software is used for data processing treatment. In particular a classical FFT (Fast Fourier Transform) software provides the amplitude and phase of the oscillating signal at the chosen harmonic. In general three periods of signal oscillations are used to perform this FFT. At frequency around $1 \mathrm{~Hz}$ more periods can be used in order to reduce the noise again. In general, the number of periods is chosen to give one data point every one minute. At low frequency (for example at frequency around 10 $\mathrm{mHz}$ ), since one period lasts more than one minute this rule is not respected anymore. The dc mean signal is obtained by an average over several periods. On one side the PXI-Rack is used for signal acquisition before data processing with Labview software. On the other side it is used to control via a NI-PXI-6534 card a home-made DAC (Digital Analogic Converter). This DAC control a current source that generates the oscillating current passing across the resistance heater on the nanocalorimetric sensor (noted $R_{C H a c}$ in Fig. 3). A Labview software triggering operation allows the synchronization in phase of the oscillating current (and tension) across the heater with the oscillating signal due to temperature oscillations. Consequently, there is a software phase synchronization of the input (power) and output (temperature). Under these circumstances, there is no supplementary phase difference between the input and the output due to the electronics chain of measurement other than that due to thermal effects. In ac-calorimetry, if the frequency of the oscillating current is noted as $F_{e l}$, then the frequency of the input thermal power is $F_{t h}=2 \times F_{e l}$ due to Joule effect. The FFT of the temperature oscillation is then made at the second harmonics $2 \times F_{e l}$ of the current frequency. The first $F_{e l}$ harmonic detection can be used to detect whether there is a dc component in the supplying oscillating current. During a thermal event or a phase transition, the small dc differential signal of the bridge can be obtained either by simple averaging over few periods of signal oscillations, or by means of the dc FFT detection. This de signal can be used, under certain conditions, to come back to the classical DSC signal. In Fig. 3, the Peltier thermo-element (Thermoelectric Cooling CP1.4-127-045L of MELCOR Thermoelectrics Company) is schematically represented with black rectangles representing the semiconducting bars. The cold face of the thermopile permits to reach a thermal bath temperature as low as $253 \mathrm{~K}$. This temperature is read with a RTD $10 k \Omega$ sensor of Heraeus company glued on one of the half-cells. A Keithley 2002 DVM (Digital Volt Meter) is used for that purpose in four-wires measuring mode. A power supply Keithley 2024 is used to generate current up to the intensity of $3 \mathrm{~A}$ across a $30 \mathrm{~W}$ heater of the Hotwatt Company in order to control the temperature of the bath in scanning or isothermal mode. Labview software with numerical PID control loop toolkit is used with GPIB bus connection to manage the two Keithley instruments. The control of the underlying scanning bath temperature rate during a measurement is independent (managed with a second computer) on the PXI generation and acquisition system. Temperature scans comprised between $0.1 \mathrm{~K} / \mathrm{min}$ to $10 \mathrm{~K} / \mathrm{min}$ can be obtained with high accuracy. Temperature range starts from 253 $K$ up to $423 K$. During a measurement the dc tempera- 


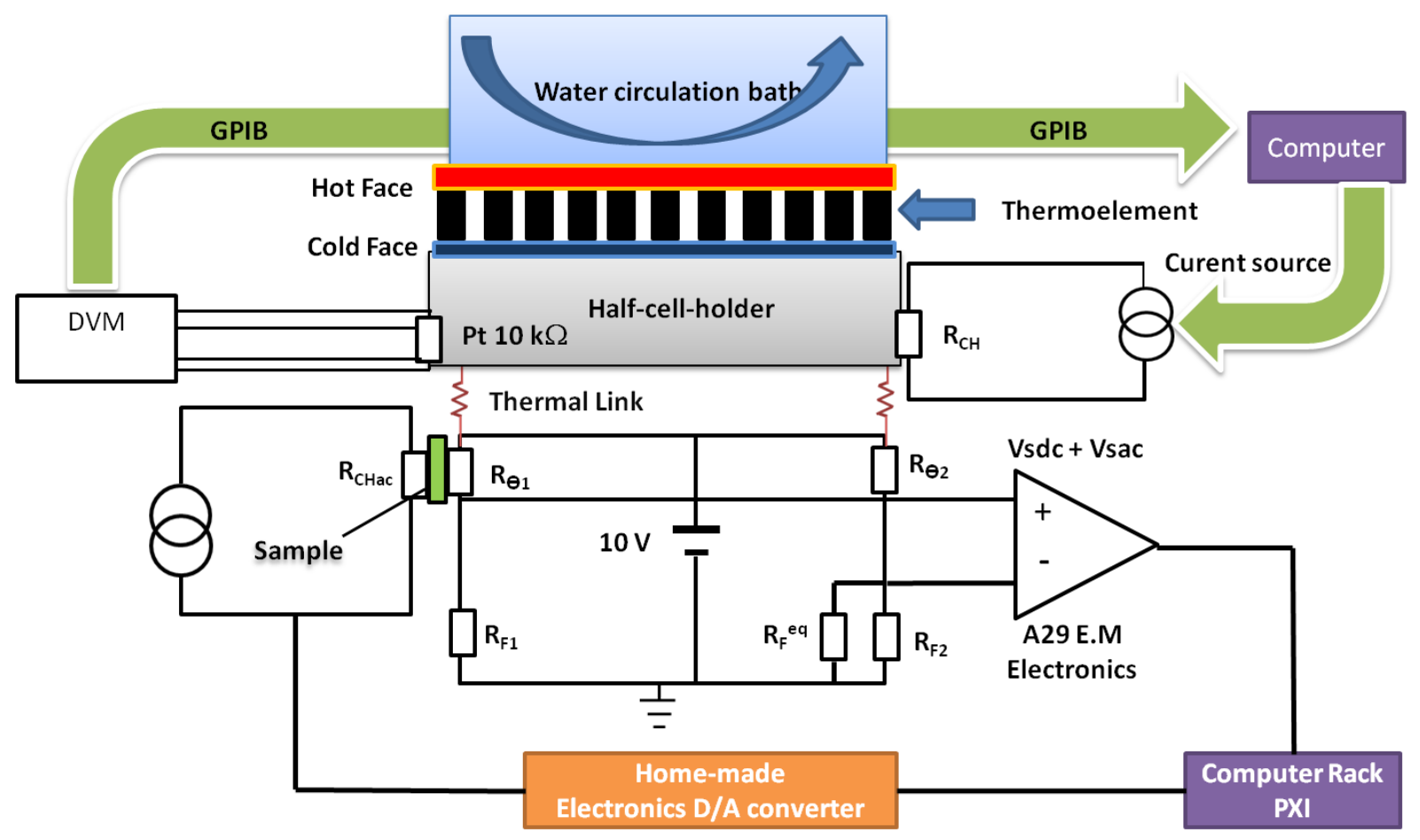

FIG. 3: Drawing of the electronic chain of measurement

ture is regulated up to few $0.1 m K$ (peak to peak). The direct reading of the voltage at the high or low output of the bridge $\left(V_{H i}\right.$ or $\left.V_{L o}\right)$ permits to access to the absolute dc temperature of the sample given by the dc component of $R_{\Theta 1}$. The calibration of the thermometers is simply carried out by means of a preliminary step in putting a standard Pt thermometer (Heraeus company) in thermal contact with the $\mathrm{Pt}$ thin film of the sensor, the dc temperature being regulated at different values. This simple calibration gives a temperature precision and a temperature slope precision of the thin film thermometer of only few percents. This implies a sample specific heat precision of only few percent. The absolute accuracy of the specific heat of the sample is limited by this calibration, by the calibration of the electronic chain, by the accuracy in the measurement of the sample mass and the mass addenda. However, it is of interest to precise here that the principal advantage of the ac-nanocalorimeter is its very high sensitivity (resolution in sample heat capacity of 10 parts per million; see next sections), high stability and high repeatability in the measurements.

\section{Thermal embodiment of the calorimeter}

The thermal embodiment of the calorimeter is depicted in Fig. 4. The calorimeter is constituted by an external stage in stainless steel. This stage defined an enclosure under vacuum because it is positioned directly on a turbo pumping device. At the top of the Fig. 4 the stainless steel wall has plenty of holes across which hermetic connectors allow electrical connections between the interior and the exterior of the calorimeter. These connectors are mostly of BNC types, but for dc tension measurements they could be of LEMO types which have less EMF (electromotive force). There are also four hermetic connectors for water and gas circulation inside the calorimeter. In this stage, the mechanical embodiment allows the location of a Peltier thermo-element with the hot face in contact to a water exchanger, and the cold face to a heat sink in copper (the end of this heat sink constitutes the copper half-cell holder such as described previously). This heat sink allows the transportation of heat between the cold source and the copper cell. A spring produces a force that maintains the TEM in high thermal contact with the water exchanger on one face and the heat sink on the other face. A thermal shield in copper surrounds the enclosure of the nanocalorimetric cell. This shield is in thermal contact with the water heat exchanger. Its temperature is regulated up to few ten's of mK (by means of a circulating water bath Julabo MW-12) ensuring a rather stable thermal surroundings for the cell, and attenuating heat loss effect by radiation between the cell and the exterior of the calorimeter. The nanocalorimetric cell is mounted and screwed on the copper half-cell holder. The electrical connections between the hermetic connectors and the top of the stainless steel stage remain fixed, while when the cell is mounted in the calorimeter, 




FIG. 4: Thermal embodiment of the calorimeter

electrical connections between this level and the cell electrical contacts have to be made using classical tin/lead welding. Copper or constantan wires of different diameters are used depending on the type of signals which have to be measured.

\section{Temperature regulation loops}

During an experiment there are different loops of temperature regulation:

1. Firstly, the temperature of the room is controlled up to $1 K$ by means of an air-conditioner.

2. Secondly, the temperature of the hot face of the Peltier thermoelement is controlled by a water bath (Julabo MW-12). The purpose of this circulating water bath is twofold:

-to evacuate the amount of heat generated at the hot face of the thermo-element

-to regulate up to few ten's of mK the hot face of the thermo-element and the corresponding thermal shield connected to it.

3. Thirdly, the cold face of the Peltier thermoelement is regulated using a power supply (Model LFI3751of Wavelength Electronics company) with integrated PID control. This cold face is then regulated up to few $m K$.
4. Fourthly, the temperature of the copper half-cell is controlled (scanning or isothermal mode) by means of a heater/thermometer couple up to few-tenth of $m K$ such as described previously.

5. Fifthly, a second circulating water bath, identical to the first one, is used to regulate the temperature of a box (in copper with heat exchangers and thermal insulation from the surroundings) of about $1 \mathrm{~m}^{3}$ of available volume. The rack PXI and all the home-made electronics (preamplifier, current sources, A/D and D/A converters, box with fixed resistors of the bridge) are enclosed inside this box. Under these circumstances, the thermal drift of all the electronic components due to temperature variations of the room is reduced by about a factor of one hundred during an experiment.

\section{THE AC-CALORIMETRY TECHNIQUE}

The principles of ac-calorimetry

Let us consider in Fig. 5 the scheme depicting a thermal system composed of a heat capacity $C$ and temperature $T$ connected by means of a thermal conductance $K_{b}$ to a thermal bath of temperature $T_{0}$. In a first time, the system is considered homogenous in temperature. The heat capacity $C$ is the sum of the addenda heat capacity (sample holder, thermometer, heater, ....) and the sample heat capacity, $C=C_{a d}+C_{s}$. A thermal power $P(t)=P_{0}(1+\cos \omega t)=P_{d c}+P_{a c}$ consisting of a dc and an ac term is supplied to the system by the heater. As a consequence, in linear regime, the temperature that is recorded by the thermometer is the sum of two contributions $T=T_{d c}+T_{a c}$. $T_{d c}$ is the mean dc temperature of the system. In stationary regime we have $\Delta T_{d c}=T_{d c}-T_{0}=P_{0} / K_{b}$, which is just the expression of the Fourier's law. $T_{a c}=\delta T_{a c} \cos (\omega t-\varphi)$ is the oscillating part of the system's temperature. $\delta T_{a c}$ is the amplitude (half-amplitude) of the temperature oscillation and



FIG. 5: Thermal drawing of the ac-nanocalorimetric cell 
$\varphi$ the phase shift between the oscillating power and the recorded oscillations. When the experimental device fulfills particular requirements, it can be demonstrated that the amplitude of the temperature oscillation is inversely proportional to the heat capacity of the system:

$$
\delta T_{a c}=\frac{P_{0}}{\omega C}
$$

As for the phase shift it is very close to $\pi / 2$. These specific requirements are the condition of adiabaticity and the condition of temperature homogeneity inside the system. The former implies that the period of temperature modulation $T_{e}=2 \pi / \omega$ has to be smaller than the relaxation time $\tau=C / K_{b}$. Physically it means that there is no oscillatory heat loss over one period of the temperature cycle $(\omega \tau>>1)$. The latter implies that the period of temperature modulation has to be greater than the internal relaxation time $\tau_{\text {int }}$ taking into account the geometry of the sample, its thermal diffusivity and all the thermal contacts which can limit the heat diffusion inside the whole system $\left(\omega \tau_{\text {int }}<<1\right)$. Physically it means that all the part of the system follows without delay the temperature cycle, i.e. that the temperature is homogeneous in all parts of the system at any time during the temperature cycle. Experimentally, we can control whether these two draconian requirements are fulfilled during the functioning of the nanocalorimeter. The temperature oscillation is recorded (amplitude and phase) at constant dc temperature as a function of the frequency. The plot of $\delta T_{a c} \times \omega=f(\omega)$ must reproduce a plateau while for the phase $\varphi=f(\omega)$ must reproduce a smooth decreasing with a range of constant values closes to $\pi / 2$ (see Fig. 6). At low frequency heat is lost towards the bath, while at high frequency there is a delay in the amount of oscillatory heat diffusing inside the sample and the device. As can be seen in the up graph in Fig. 6, the frequency range which fulfills the requirements is limited up to $10 \%$ between about $50 \mathrm{mHz}$ and $1 \mathrm{~Hz}$. It is less visible for the phase. However, the measurement of the frequency response as well as the use of a thermal model of the nanocalorimeter can be used to enlarge the working frequency range between $8 \mathrm{mHz}$ and $3 \mathrm{~Hz}$ (see a next section). The accuracy and resolution are smaller at the boundaries of the range because corrective terms like the thermal conductance $K_{b}$ or the thermal conductivity of the sample have to be accurately known in order to be able to extract correctly the complex heat capacity of the sample itself and its variations along time. It has to be pointed out that this is what is usually done in other dynamic calorimetric methods like in TMDSC (knowledge of $K_{b}$ for low frequencies measurements) or like in the three-omega method (knowledge of the sample thermal conductivity and thermal contacts for high frequencies measurements).
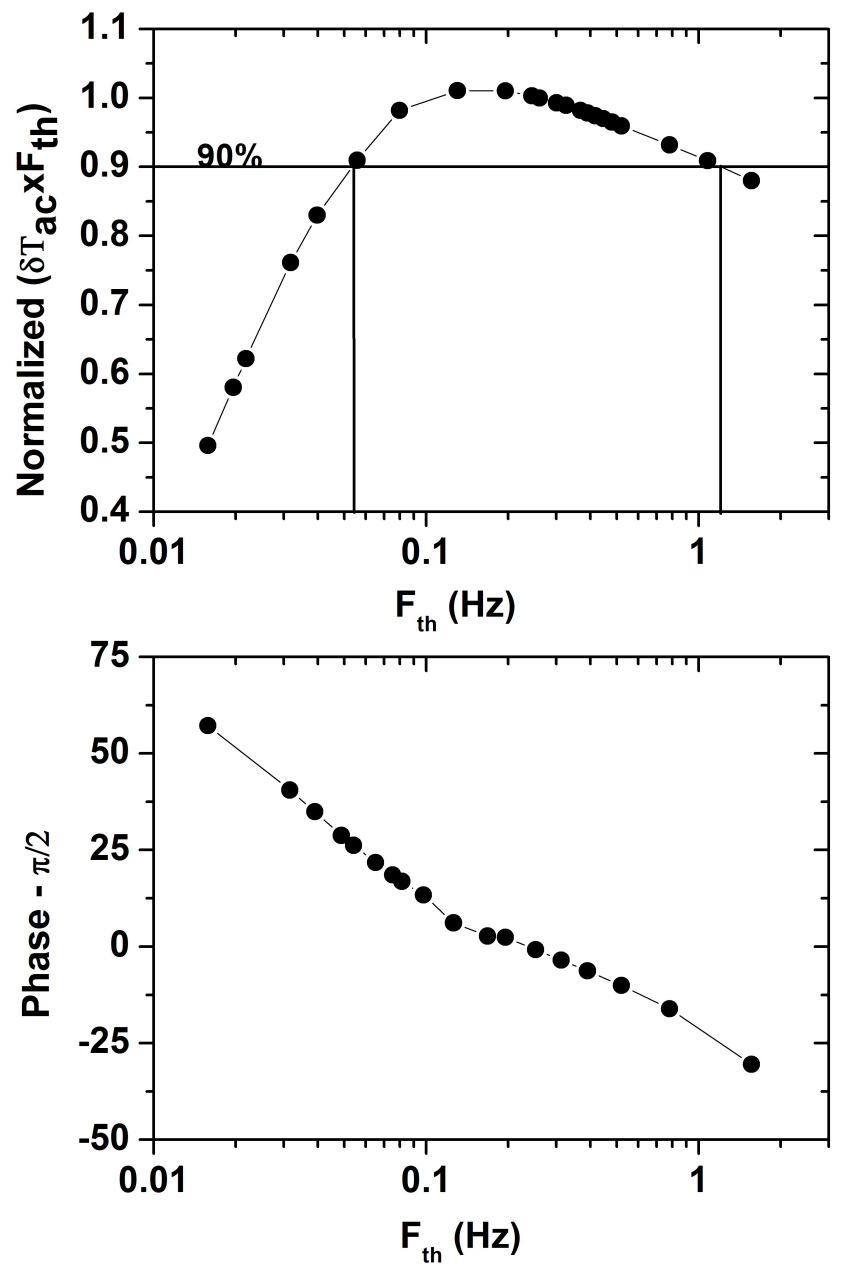

FIG. 6: Normalized amplitude and phase of the oscillating temperature as a function of the frequency. Black lines are just an indication for enlightening of the frequency behavior.

The non-equilibrium aspect of the ac-calorimetry: frequency dependent complex heat capacity

As either the amplitude or the phase of the temperature oscillation are recorded, a complex susceptibility can be defined such as follows:

$$
C^{*}=\frac{P_{a c}}{i \omega T_{a c}}=C^{\prime}-i C^{\prime \prime}
$$

The physical meaning of this complex quantity has been discussed in Ref. [2, 8] and references therein. Like in dielectric spectroscopy, the real part of the complex heat capacity gives insights on the predisposition of the system to respond or not to the corresponding excitation. For dielectric spectroscopy the degrees of freedom involved are the electrical dipoles. For calorimetry, they are all the degrees of freedom involved in the energy exchanges between the system and the surroundings. We speak about frozen-in structural degrees of freedom for glasses while 
these degrees of freedom are free to move and participate to the measured heat capacity for the corresponding super-cooled liquid. As for the imaginary part, it gives insights on the maximum of the relaxation of these internal degrees of freedom involved in the thermal process. The imaginary part of the complex heat capacity in oscillating calorimetry is always connected to the net entropy produced over one period of the temperature cycle which can take place during a phase transition or whatever thermal events under interest occurring out of equilibrium $[2,8,9]$. In ac-calorimetry it is also connected to irreversible processes that can affect the temperature of the system due to certain thermal characteristics of the device [9]. When time-lag effects (phase shift) due to experimental relaxation times are removed using an appropriate thermal model, the complex heat capacity must be written for example such as:

$$
C^{*}=\frac{C^{e q}-C_{\infty}}{1+i \omega \tau}
$$

where in this equation a physical process with a simple relaxation time $\tau$ has been considered (Debye relaxation). The difference $C^{e q}-C_{\infty}$ in the numerator is the difference between the equilibrium heat capacity (classical static heat capacity where all the degrees of freedom participate to the measured temperature elevation), and the heat capacity defined by the infinitely fast degrees of freedom only (the other ones having being frozen-in and not participating to the measured temperature change). In the case of the glass transition, however, a distribution of relaxation times must rather be considered which modifies in general the previous expression of the complex heat capacity.

\section{OSCILLATING SIGNAL}

\section{Resolution of the device}

The ac-calorimetry is a highly sensitive method of measurement of the heat capacity. The resolution in the measured heat capacity $\Delta C_{p} / C_{p}$, i.e. the availability to detect a small variation of the heat capacity on the measured total heat capacity, corresponds to the inverse of the signal to noise ratio. The physical signal $T_{a c}$ (temperature oscillation) depends on the mean power $P_{0}$ dissipated in the heater resistance, on the absolute value of the measured heat capacity, and on the frequency such as given in Eq. (1) (at least on the adiabatic plateau). The final oscillating signal measured by the transducer is a voltage obeying to the following law:

$$
V_{a c}=A(T) V_{0} \alpha T_{a c}
$$

where $V_{0}$ is the voltage polarization of the Wheatstone bridge, $\alpha$ is the temperature coefficient of the resistive thermometer, and $A(T)$ is a coefficient without dimension that depends on temperature. $A(T)$ is a function depending on the values of all the resistors involved in the Wheatstone bridge. This oscillating signal is recorded as a function of time. A numerical FFT is carried out in order to extract the amplitude and phase at a given frequency. Under these circumstances, with the use of appropriate high and low pass filters, the noise bandwidth is reduced to its minimum limit. When the electronic chain of measurement is adapted to the impedance of the thermometric sensor, this limit is given by the white noise (Johnson noise) of this resistive sensor taken on this frequency bandwidth $V_{N}=\sqrt{4 k_{B} T R_{N} \triangle f}$, where $k_{B}$ is the Boltzmann constant, $T$ the absolute temperature, $\triangle f$ is the frequency bandwidth over which the measurement is carried out (filter, averaging, etc...), and $R_{N}$ the noise equivalent resistance of the Wheatstone bridge polarized by the constant voltage $V_{0}$. The signal to noise ratio is consequently given by:

$$
\frac{S}{N}=A(T) \sqrt{\frac{P_{w}}{P_{N}}} \alpha T_{a c}
$$

where $P_{w}=V_{0}^{2} / R_{N}$ is the power dissipated in the Wheatstone bridge and $P_{N}=V_{N}^{2} / R_{N}$ is the noise power in the frequency bandwidth $\Delta f$. As a consequence, for a given power dissipated in the bridge and for a given power dissipated in the heater, the resolution on the heat capacity measurement depends only on the temperature coefficient of the thermometer. The maximum suitable power dissipated in the bridge (polarization tension $V_{0}$ ) is physically limited by the maximum suitable power dissipated in the thermometric sensor, itself limited by the overheating effect. The maximum suitable power dissipated by the heater (power $P_{0}$ ) is limited by the overheating effect and the maximum suitable value for the amplitude of temperature oscillation regarding the physics (e.g. temperature width of phase transitions) to be probed. A second point is that for an oscillatory signal, the so called parasitic electromotive forces (EMF: Seebeck effect) are removed. Moreover, the thermal drift of the dc baseline temperature is also of lesser influence on the measured oscillating signal than for usual dc temperature signals like in DSC for example. Finally, around a constant dc temperature, the oscillating signal can be averaged (reduction of the frequency bandwidth) over rather long time, increasing again the signal to noise ratio. A point of very high importance is the reduction of the addenda heat capacity. Indeed, the smaller the addenda heat capacity with respect to the sample heat capacity, the higher the ability to detect a fine variation in the heat capacity of the sample. In that case, the use of microfabrication technologies allowing the development of micro-sensors of very low mass is of very high interest. Such as discussed in a previous section, the addenda heat capacity of the sensor participates to less than $50 \%$ of the total measured heat capacity. The resolution in the sample heat 
capacity (without addenda) is consequently only reduced by a factor of two. For the measurement of the absolute real value of the sample specific heat (accuracy), it depends on the care that is taken to calibrate the different parts of the electronic chains, on the calibration of the thermometer, on the precision on the mass of the measured samples, on the mass of the addenda, and on the precise control of the phase of the oscillating signal. An example of high absolute accuracy in membrane-based ac-calorimetry is given in reference [33] while high resolution is well described in [24]. In our case, we have mostly chosen to concentrate our effort on the resolution rather than on the absolute accuracy. The temperature coefficient of the sputtered platinum thermometer is on the order of $2.5 \times 10^{-3} K^{-1}$. Thin films of semi-conducting material can have about ten times higher temperature coefficients. However, platinum is known to be one of the more stable metallic materials for thermometry, particularly for resistive thin films thermometry. We thus have focalized on this metallic material in choosing as a priority the stability than the absolute accuracy. This choice is due to the nature of the amorphous materials that we want to measure being non-equilibrium systems slowly evolving along time. Performances of the device in terms of resolution and repeatability are discussed in a next section.

\section{THERMAL MODEL OF THE DEVICE}

\section{Extraction of $C^{\prime}$ and $C^{\prime \prime}$ on enlarged frequency range}

As shown on graphs in Fig. 6, the oscillating response of the transducer depends directly on the nanocalorimetric membrane based micro-sensor, and how heat propagates within the different parts of the device between the heater and the thermometer. A thermal model has been developed in order to extract the real and imaginary components of the heat capacity of the sample itself from the amplitude and phase of the oscillating temperature of the entire device in the widest possible frequency range. The model is extended in the whole domain of temperatures of the experiments. The exact calculation concerning this model will be detailed in a forthcoming publication. We briefly describe here the principal steps. The device is considered as a superposition of three media of different thermal conductivities and specific heats such as depicted in the drawing of Fig. 7. The equation of heat propagation in these different media in cylindrical coordinates is given by:

$\frac{\partial^{2} T(r, z, t)}{\partial z^{2}}+\frac{\partial^{2} T(r, z, t)}{\partial r^{2}}+\frac{1}{r} \times \frac{\partial T(r, z, t)}{\partial r}-\frac{1}{D_{i}} \times \frac{\partial T}{\partial t}(r, z, t)=0$

where $D_{i}=k_{i} / \rho_{i} c_{i}$ is the thermal diffusivity of the medium $i$, with $k_{i}$ the thermal conductivity, $c_{i}$ the spe-

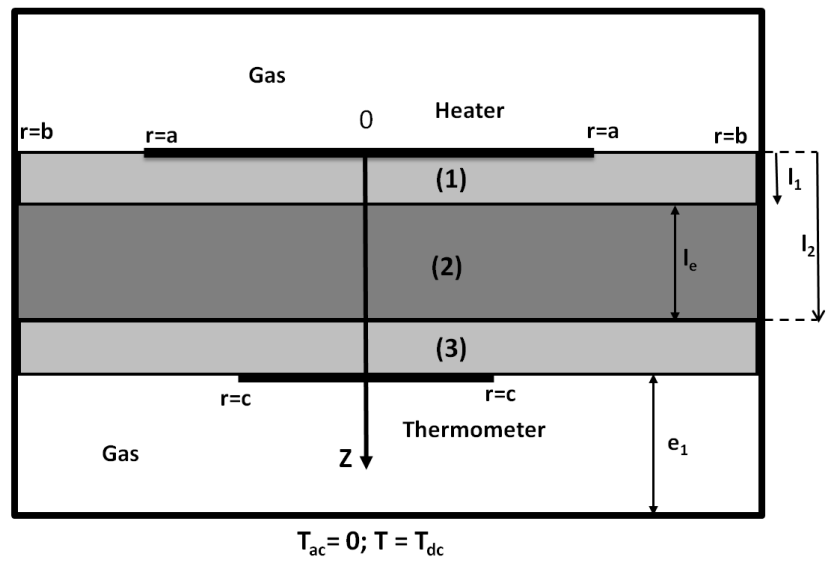

FIG. 7: Thermal modeling of the nanocalorimetric cell with three different media (1), (2) and (3).

cific heat, and $\rho_{i}$ the density. Using the variable separation principle $T(z, r, t)=T_{1}(t) \times T_{2}(z, r)$, the general solution of this equation is:

$$
T_{1}(z, r)=\sum_{n=1}^{\infty}\left(A_{1} \cosh \left(\mu_{1} z\right)+B_{1} \sinh \left(\mu_{1} z\right)\right) J_{0}\left(\alpha_{n} r\right)
$$

where the $\alpha_{n}$ with $n$ the summation index are determined by the boundary equation $J_{0}\left(\alpha_{n} b\right)=0$ at the distance $b$ where there is no temperature oscillation. $\mu_{1}$ of the medium 1 is determined from the $\alpha_{n}$ by means of the relation $\mu_{1}^{2}=\alpha_{n}^{2}+i \omega / D_{1}$. The second boundary equation is given at the level of the heater by mean of the relation $P_{0} / \pi a^{2}=-k_{1}\left(\partial T_{1} / \partial z\right)_{z=0}$ where $P_{0} / \pi a^{2}$ is the mean power per units of surface dissipated by the heater on all the surface of the membrane delimited by the heater. On the second medium (medium 2) we have relations such as $\mu_{2}^{2}=\alpha_{n}^{2}+i \omega / D_{2}$, and the boundaries conditions at the distance $z=l_{1}$ between medium 1 and 2 are $T_{1}=T_{2}$ and $\left.\left.-k_{1} \partial T_{1} / \partial z\right)_{z=l_{1}}=-k_{2} \partial T_{2} / \partial z\right)_{z=l_{2}}$. We have the same type of boundaries conditions at the distance $z=l_{2}$ between the medium 2 and 3 . This gives us at the level of the thermometer (distance $l$ ) the following expression of the complex temperature integrated on all the surface $\pi c^{2}$ of the second membrane delimited by the thermometer:

$$
\begin{gathered}
T_{3}(l)=\sum_{n=1}^{\infty} \frac{4 P_{0}}{K_{1} \pi a b^{2} c} \frac{J_{1}\left(\alpha_{n} a\right) J_{1}\left(\alpha_{n} c\right)}{\alpha_{n}^{2} J_{1}^{2}\left(\alpha_{n} b\right)}\left\{\sinh \frac{\mu_{2} l_{e}}{2} \times\right. \\
{\left[\left(\frac{\mu_{2} k_{2}}{\mu_{1} k_{1}}+\frac{\mu_{1} k_{1}}{\mu_{2} k_{2}}\right)\left(\beta_{1} \sinh 2 \mu_{1} l_{1}+\mu_{1} \cosh 2 \mu_{1} l_{1}\right)+\right.} \\
\left.\left.\mu_{1}\left(\frac{\mu_{2} k_{2}}{\mu_{1} k_{1}}-\frac{\mu_{1} k_{1}}{\mu_{2} k_{2}}\right)\right]+\cosh \mu_{2} l_{e}\left(\beta_{1} \cosh 2 \mu_{1} l_{1}+\mu_{1} \sinh 2 \mu_{1} l_{1}\right)\right\}
\end{gathered}
$$

This solution allows fitting the measured experimental frequency behaviors of the amplitude and phase of the 
oscillating temperature with a good accuracy. At this stage, the remaining question is how can we extract the interesting physical information on the PVAc sample from this temperature oscillation (amplitude and phase)? More precisely, we would like to obtain by means of this model the real and imaginary components of the complex heat capacity of the PVAc sample at every accessible frequency and on all the temperature range during various experimental protocols. For that purpose an analytical calculation valuable only for the low frequency domain (which is a correct assumption for the working frequency range used between $8 \mathrm{mHz}$ and $3 \mathrm{~Hz}$ ) yields to (integrated on the surface $\pi c^{2}$ delimited by the thermometer):

$$
\left.\frac{\omega T_{3}(l)}{P_{0}}=\frac{\omega}{\pi a^{2} k_{1} D_{a}} \sum_{n=1}^{\infty} \frac{4 J_{1}\left(\alpha_{n} a\right) J_{1}\left(\alpha_{n} c\right) a}{\alpha_{n}^{2} J_{1}^{2}\left(\alpha_{n} b\right) b^{2} c\left(1+\frac{D_{n b}}{D_{a}}\right)}\right)
$$

where the $D_{a}$ and $D_{n b}$ are known from the equation (8). The work remaining to do is to determine $c_{2}$ and $k_{2}$ of the medium 2 (PVAc) by calculation from the measured oscillating temperature. We obviously write $c_{2}=c_{2}^{\prime}-i c_{2}^{\prime \prime}$. This could be done in several steps. Firstly it could be remarked that in this low frequency range, the modulus and phase of the whole term under the summation follow the approximations $\left|\sum\right| \sim 1$ and $\varphi\left(\sum\right) \sim 0$ respectively. Then, in the temperature range where we know that there is no imaginary part in the specific heat of the PVAc $\left(c_{2}^{\prime \prime}=0\right)$, i.e. in the glassy range and in the liquid range, we calculate up to the first order $\left|\sum\right|$ and $\varphi\left(\sum\right)$. Secondly, the term before the summation can be approximated using a series development up to a third order of the hyperbolic sinus and cosines, which correspond to terms in $\omega^{3}$. From these developments, it appears $C_{1}$ the heat capacity of medium 1 (and 3 ), $K_{1}$, the thermal conductance of medium 1 (and 3), and $C_{2}$ and $K_{2}$ the heat capacity and thermal conductance of medium 2 (PVAc). At this stage, we need to know the thermal conductivity and specific heat of the medium 1 , which are strictly the same than those of the medium 3 . Let us recall that the media 1 and 3 are the same superposition of polyimide membrane (Upilex@) and gold (see the next section explaining the conditioning of the PVAc sample in the cell). We use the assumptions that $k_{\text {upilex+gold }}=k_{\text {upilex }}$ and $c_{\text {upilex+gold }}=c_{\text {upilex }}+c_{\text {gold }}$, all these data being found as a function of temperature in the literature. To determine exactly the value of $\left|\sum\right|$ and $\varphi\left(\sum\right)$, we previously used one measurement of a polyimide (Upilex@) sample in place of the PVAc sample but without gold. It is like a calibration measurement with one reference polyimide sample. From all these steps, we obtain $C_{2}^{\prime}$. Now in the transition range where $C_{2}^{\prime \prime} \neq 0$, we obtain $C_{2}^{\prime}$ and $C_{2}^{\prime \prime}$. Let us also noticed that $K_{b}$ the thermal conductance of the heat link (given principally by the gas back to each membrane) is obtained from this calculation. Now the calculation can be adjusted for all frequencies and temperatures finally giving $c_{2}^{\prime}$ and $C_{2}^{\prime \prime}$ the real and imaginary part of the frequency dependent complex specific heat of PVAc measured upon different protocols.

\section{RESULTS}

\section{PVAc sample conditioning}

PVAc samples have been kindly given by Pr. G. McKenna. The average molecular weight is $157 \mathrm{~kg} / \mathrm{mol}$ and the polydispersity index (PDI), which indicates the distribution of individual molecular masses in a polymer, is 2.73. The samples are initially made of individual balls of about $100 \mathrm{mg}$. One ball is first put in a container under vacuum in an oven up to a temperature of $423 \mathrm{~K}$ during one night. The residual gas filled in the polymer (gas bubbles are observed at the starting of this procedure) is released by this way. The liquid sample is then seen as completely transparent without any bubbles. The temperature of PVAc is then slowly reduced to the temperature of $363 \mathrm{~K}$. At this temperature, the PVAc is of viscosity high enough allowing pressing it in a hot press in order to obtain films of about $100 \mu \mathrm{m}$ thick. Once the thin film is obtained, at ambient temperature small disks of $8 \mathrm{~mm}$ in diameter with thickness of about 100 $\mu m$ are cut with a punch. A sample is then ready for a measurement. It is taken as a sandwich between two gold disks of $10 \mu \mathrm{m}$ thick with the same diameter. The sandwich is positioned in the nanocalorimetric cell such as described in the first section.

\section{PVAC glass transition}

In Fig. 8 (up graph) the raw data of the modulus of the complex heat capacity of the PVAc sample plus the addenda is shown during a cooling and a successive heating. The modulus of the complex heat capacity is simply obtained by means of the modulus $\delta T_{a c}$ (half amplitude) of the oscillating temperature by means of the relation $\left|C_{p}^{*}\right|=P_{0} / \omega \delta T_{a c}$. The amplitude $\delta T_{a c}$ is obtained from the bridge output by means of equation (4). For the sake of simplicity, the use of this equation allows a rapid comparison with the PVAc heat capacity (for example with DSC data), but we have to bear in mind that it is raw data without any subtraction of the addenda and without any correction due to the frequency dependence of the nanocalorimetric cell. An example of correct data treatment is made in a subsequent section where the real and imaginary parts of the PVAc is calculated by means of the thermal model described above. In all the following figures, the $\left|C_{p}^{*}\right|$ is plotted with this simplified formula. In this experiment, the system is set at around $360 \mathrm{~K}$ during $20 \mathrm{~min}$ and then cooled from this temperature down to $281 \mathrm{~K}$ at $-1.2 \mathrm{~K} / \mathrm{min}$ (blue curve). It is then instantaneously heated from $281 K$ to $360 \mathrm{~K}$ 

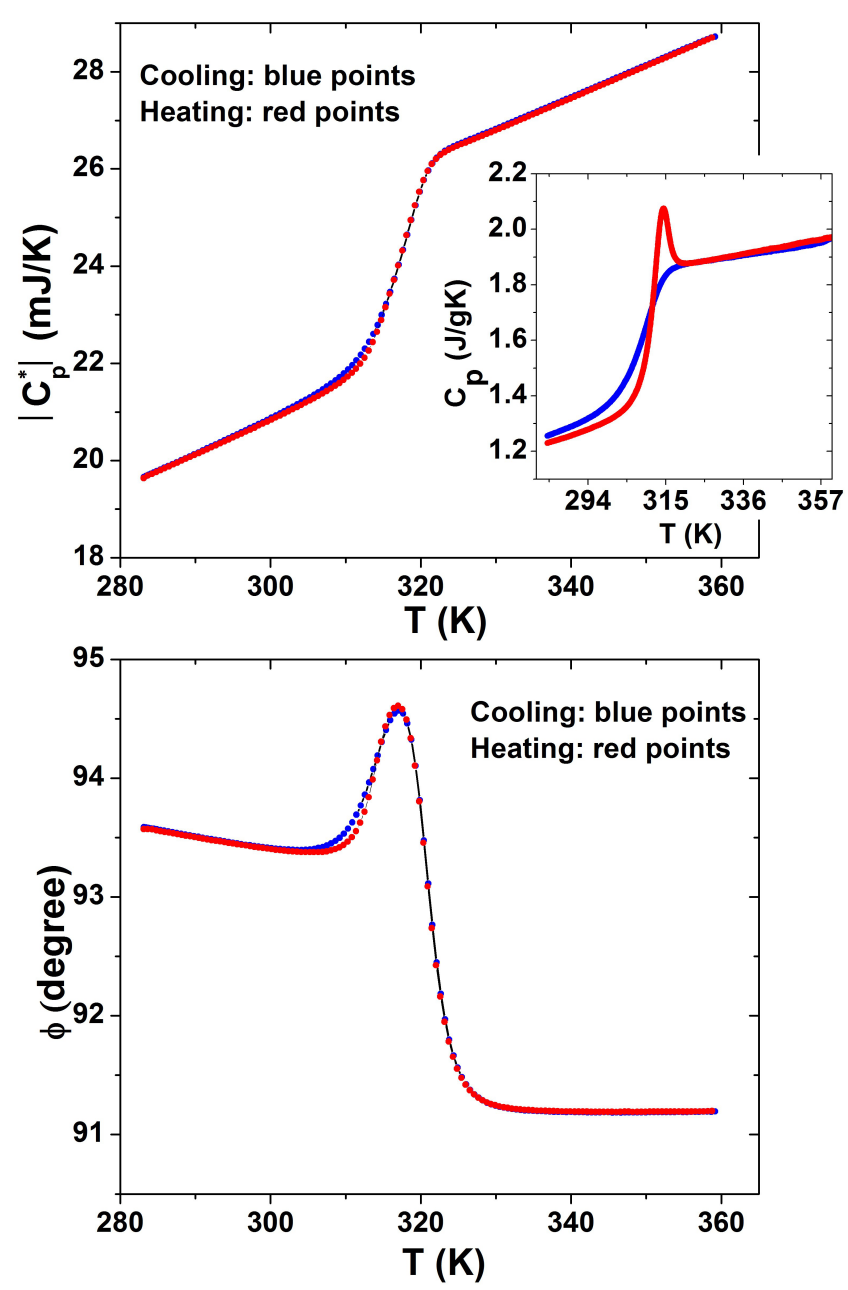

FIG. 8: Top graph: raw data of the modulus of the complex heat capacity measured during a cooling and a successive heating. Inset: specific heat of PVAc measured by DSC under the same experimental conditions. Bottom graph: phase of the temperature oscillation measured during the same conditions.

at a rate of $1.2 \mathrm{~K} / \mathrm{min}$ (red curve). The frequency is of $0.1954 \mathrm{~Hz}$ and the mean power of the heater is adjusted to give an amplitude of temperature oscillation of about $450 \mathrm{mK}$ (half amplitude). During cooling a typical jump in the heat capacity is observed which is typical of vitrification. During the successive heating the jump is also visible with a small delay due to the glass stabilization process. These two curves indicate an idea of the reproducibility of the glassy and super cooled liquid baselines between cooling and heating. Repeatability of the nanocalorimeter for different experiments upon the same experimental conditions is discussed later. If we evaluate the glass temperature $T_{g}$ in taking the inflexion point in the $C_{p}(T)$ curve during cooling, we observe that under the same experimental condition (and with the same conditioned sample) the value is higher (around 317
$K)$ with ac-calorimetry than in classical DSC. The DSC measurements (expressed as specific heat) are shown in the inset of Fig. 8. The $T_{g}$ is around $309 K$ extracted from the cooling. This $T_{g}$ drift effect is usual when these two calorimetric methods are compared in the study of a glass-former under the same experimental conditions. As an example, the $T_{g}$ of the total $\mathrm{Cp}$ is lower than that of the real part in TMDSC [13, 15, 17]. Moreover, in DSC the heating shows an endothermic peak (structural recovery) that is not visible in ac-calorimetry. This is the proof that the influence of experimental time scales are of fundamental importance in the thermodynamic measurements of the dynamic glass transition. Moreover, the stabilization effect of the glass (with a heat capacity curve during the heating below that obtained during the previous cooling and remaining in this state towards higher temperatures than during the cooling) is more pronounced in DSC than in ac-calorimetry. In the bottom graph of Fig. 8, the phase is represented upon the same conditions. The phase generally undergoes a peak during cooling and heating, but it disappears at lower frequency. The small difference between cooling and heating is also visible on these curves. These raw data on the phase are of similar shapes from that obtained by Jeong and al. on CKN glass with a sensitive ac-calorimeter [28].

\section{Resolution of the nanocalorimeter}

In order to enlighten the performances of the calorimeter, an experiment is proceeded revealing the noise of the measurement either on the modulus or on the phase of the recorded temperature oscillation. After $20 \mathrm{~min}$ of stabilization at $363 \mathrm{~K}$, the sample is cooled down to 339 $K$ at a rate of $-1.2 \mathrm{~K} / \mathrm{min}$. The dc temperature is regulated at this value during few hours while the oscillating temperature is recorded. At this temperature, above the calorimetric $T_{g}$, the system still undergoes a stabilization process due to the presence of a relaxation time although this effect is undeniably more pronounced below $T_{g}$. This stabilization process is seen as a small and slow decrease of the heat capacity (modulus) and the phase along time (see the two inserts in Fig. 9). The physical interpretation of this stabilization process will be the topic of a forthcoming publication. It has however already been discussed by Tombari and al. on the complex heat capacity on TPTG-ether [34]. A ninth order polynomial fit (seen as black lines in the inserts in Fig. 9) is subtracted enlightening the noise on the modulus of $C_{p}^{*}$ and on the phase during this isothermal process (see Fig. 9). The peak to peak noise $\Delta\left|C_{p}^{*}\right|$ is of about $500 \mathrm{~nJ} / \mathrm{K}$. The resolution of the experiment is obtained in dividing this peak to peak noise by the absolute value of the heat capacity of the system at each time. In Fig. 9, the resolution on the modulus and on the phase are directly represented in $\%$ on the same scale for comparison. While for 


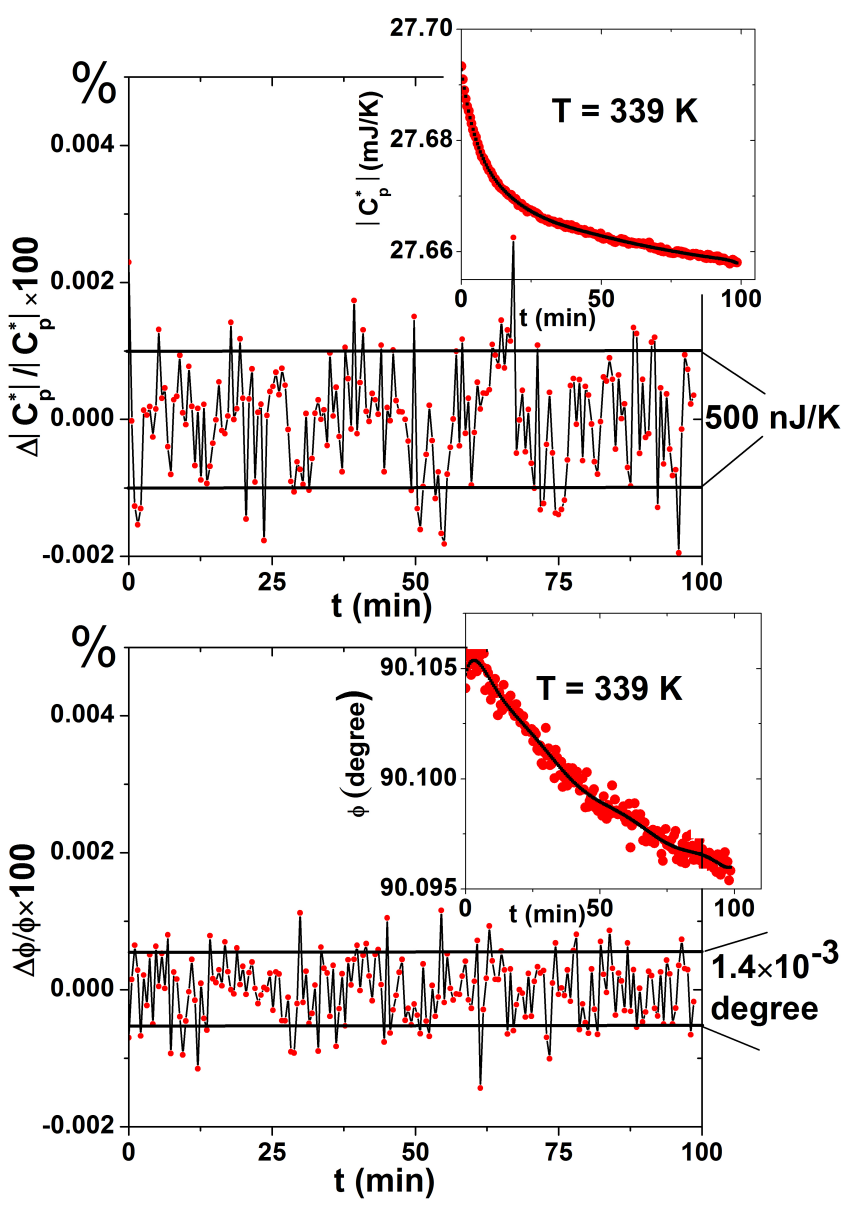

FIG. 9: The top and bottom graphs represent the resolution of the nanocalorimeter either on the modulus of the complex heat capacity or on the phase of the temperature oscillation. In the two graphs the resolution is given in percentage on the same y-scale. The two corresponding insets show the raw data of the modulus of the complex heat capacity and the phase of the temperature oscillation measured as a function of time during a constant temperature regulation of $100 \mathrm{~min}$

the modulus of $C_{p}^{*}$ it is of the order of $\Delta\left|C_{p}^{*}\right| /\left|C_{p}^{*}\right| \sim \pm$ $10^{-5}$, for the phase it is merely two times better with $\Delta \phi / \phi \sim \pm 5.10^{-6}$. These values are obtained on a bandwidth of the order of $1 \mathrm{~min}^{-1}$, because the amplitude and phase of the oscillating temperature are calculated with a fast Fourier transform taken on a number of periods spread on a time scale of about $1 \mathrm{~min}$. In other words, it is thus possible to detect heat capacity changes during thermal events just higher than 20 parts per million of the total measured heat capacity, and phase changes just higher than 10 parts per million of the measured phase. Obviously, these values can be slightly different depending on the experimental conditions (frequency, oscillating power, bandwidth of the FFT, dc temperature rates, stability of the room temperature, etc...). In order to emphasis the importance of such high resolution, it can be remarked that with classical commercial TMDSC the slow decreases visible in the insets in Fig. 9 would not have been recorded. Indeed, for the modulus for example, the decrease is of about $40 \mu \mathrm{J} / \mathrm{K}$, which correspond to a relative variation of about one part per thousand. Knowing that TMDSC have resolution around few percent, the decrease will be hidden in the noise of the apparatus.

\section{Repeatability of the results}

In the study of soft-matter, it is known that the measurement of a physical property at different times never provides exactly the same value. This is particularly true in the study of slowly relaxing systems such as glasses for which the physical properties depends on the history of the systems. Moreover, certain systems such as polymeric glass-formers undergo slow temporal evolution of their properties linked to chemical degradation or chemical interaction with the surroundings. This is also our case here where two measurements made under the same experimental conditions but at different periods of time give slightly different results. In particular the PVAc glass former is known to have its glass transition properties which depend on the quantity of water absorbed [35]. During about two years of experiments, the sample has been put different times under ambient atmosphere and then pumped again under vacuum. We remarked that this slow absorption or desorption of water produces a slight drift of the glass temperature $T_{g}$. However, in Fig. 10 and 11, two identical experiments carried out at few days of interval are shown in order to enlighten the typical repeatability of the results with the nanocalorimeter. In each figure the modulus of the complex heat capacity and phase of the oscillating temperature are shown as a function of temperature. The Fig. 10 and 11 depict two different experiments. In each figure, two experiments made under the same experimental conditions are shown but there are carried out at two days of interval between each. In Fig. 10, the experiments consist firstly in a cooling from about $363 K$ (temperature at which the systems remained during $20 \mathrm{~min}$ ) at a rate of -1.2 $\mathrm{K} / \mathrm{min}$ towards about $283 \mathrm{~K}$. The thermal frequency is of $0.1954 \mathrm{~Hz}$. The mean power $P_{0}$ provides a temperature oscillation (half amplitude) of about $450 \mathrm{mK}$. In Fig. 11, the two experiments consist in a heating at a rate of $0.2 \mathrm{~K} / \mathrm{min}$ measured just after the previous cooling shown in Fig. 10 from about $283 K$ toward about 363 $K$. At this scale the repeatability of the results seem of high quality with a difference between the two curves of about only few $\mu J / K$ in the supercooled liquid state. In the insets of Fig. 10 and 11 a zoom of the curves is focalized inside the glass transition range. On the modulus, a small drift $\triangle T_{g} \sim 0.2 \mathrm{~K}$ of the glass temperature is observable for the two coolings while a small drift $\triangle T_{g} \sim$ $0.15 K$ is observable between the two heatings. For the 

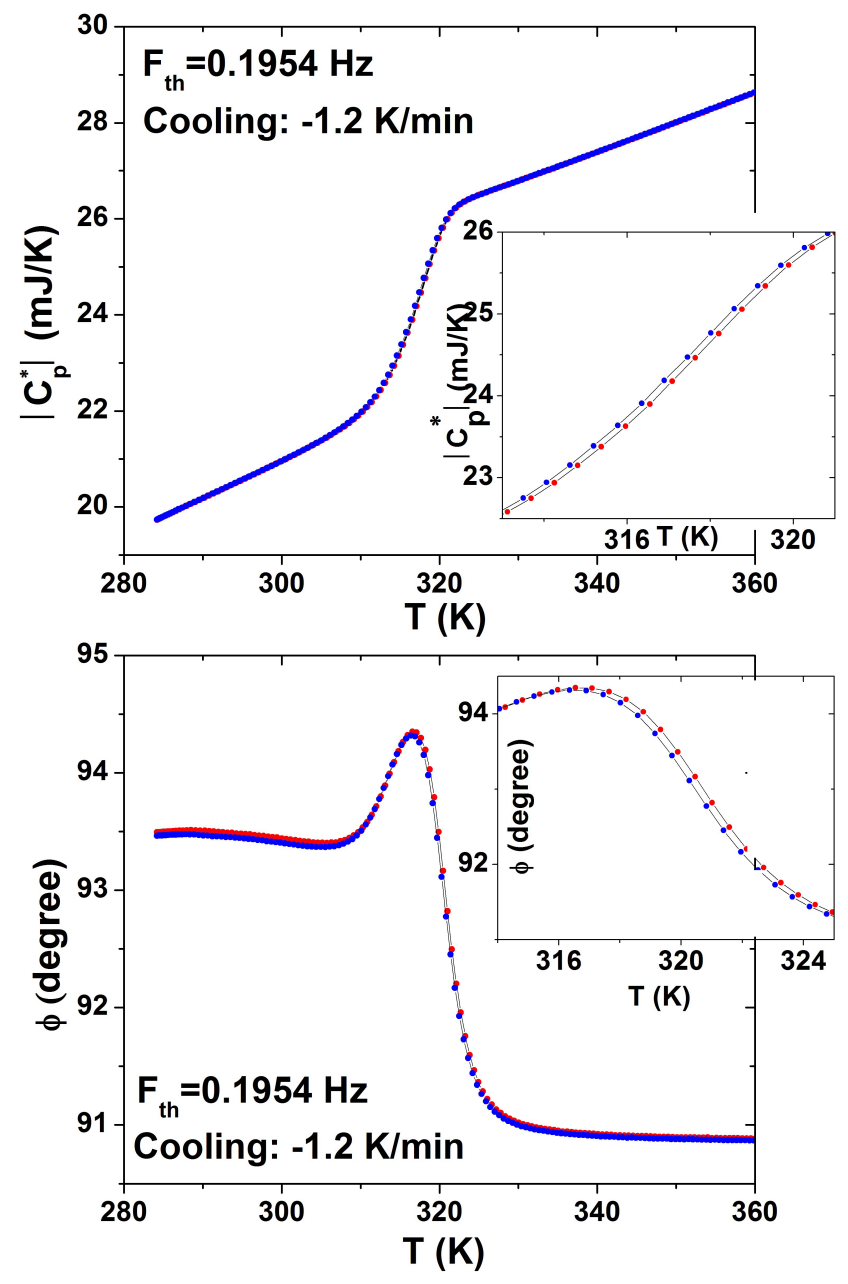

FIG. 10: The top and bottom graphs are the raw data of the modulus of the complex heat capacity and the phase of the temperature oscillation measured during two cooling ramps at the same rate, the two experiments being made at two days of interval. The two corresponding insets show a zoom in the temperature range where the sample vitrifies in order to enlighten the repeatability of the measurements.

phases, a vertical and horizontal drifts are visible. In insets, we focalize on the identical $T_{g}$ drifts discussed for the modulus, but a vertical drift axis is also visible either in the glassy state or in the super-cooled liquid state. As an typical example, for the two heatings at $0.2 \mathrm{~K} / \mathrm{min}$ a difference of about $\triangle \Phi \sim 15 \times 10^{-3}$ degree is visible in the supercooled liquid state while it is about two times greater in the glassy state. These small differences could occur if $20 \mathrm{~min}$ of waiting time at $363 \mathrm{~K}$ is not enough to erase completely the memory of all the history of the system between these two days of interval. As a conclusion of this section, the physics that could be extracted from the measurements made with this nanocalorimeter must produce effects larger than the small drifts just discussed above. We would also point out that repeatability


FIG. 11: The top and bottom graphs are the raw data of the modulus of the complex heat capacity and the phase of the temperature oscillation measured during two heating ramps at the same rate, the two experiments being made at two days of interval. The two corresponding insets show a zoom in the temperature range where the sample undergoes structural recovery in order to enlighten the repeatability of the measurements.

of measurements on complex systems such as glasses or biological molecules are only rarely discussed in the literature.

\section{Complex specific heat of PVAc}

Using the thermal model described in a previous section, the real and imaginary part of the complex specific heat of PVAc can be computed. They are shown in Fig. 12 along with the specific heat of PVAc measured by DSC. The read line corresponds to the specific heat of PVAc measured by DSC during a heating at a rate of $1.2 \mathrm{~K} / \mathrm{min}$ after a cooling at a same rate. The 


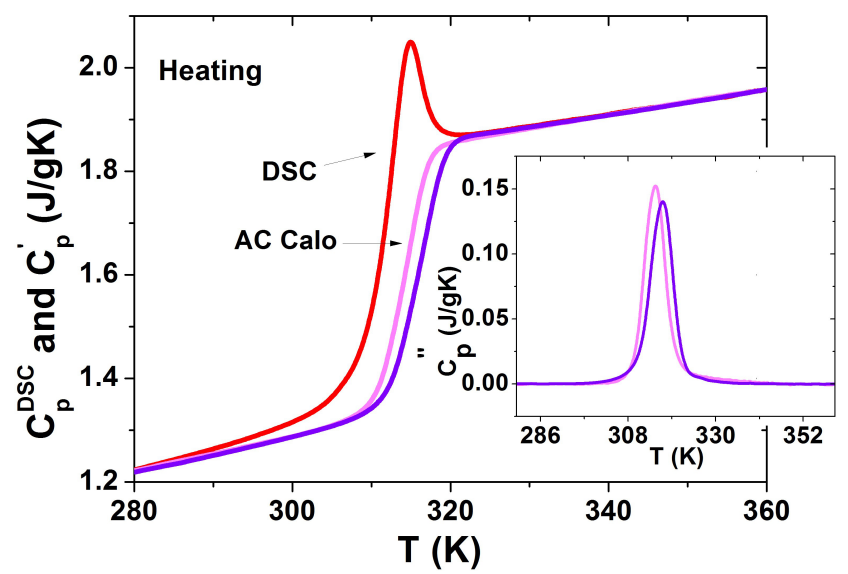

FIG. 12: The specific heat of PVAc as measured by DSC as a function of temperature is compared with the real parts of the specific heat of PVAc calculated at two different frequencies obtained during different heating ramps (see text for details). In the inset, the two corresponding imaginary parts of the complex specific heat of PVAc are shown.

magenta line corresponds to the specific heat of PVAc (real part) calculated from the measurements with the ac-nanocalorimeter at a frequency of $31.6 \mathrm{mHz}$ during a heating at a rate of $0.2 \mathrm{~K} / \mathrm{min}$ just after a cooling at the same rate. The violet line corresponds to the specific heat of PVAc (real part) calculated from the measurements with the ac-nanocalorimeter at a frequency of $0.126 \mathrm{~Hz}$ during a heating at a rate of $1.2 \mathrm{~K} / \mathrm{min}$ just after a cooling at the same rate. In inset, the imaginary parts of the complex heat capacity of PVAc are shown for the two previous modulated experiments. These figures show that it is possible to extract from the thermal model the physical quantities $c_{p}^{\prime}$ and $c_{p}^{\prime \prime}$ which can be directly compared to the specific heat measured with DSC. It can be easily observed how the $T_{g}$ depend on the frequency and experimental conditions, and how the maximum and amplitude of the $c_{p}^{\prime \prime}$ peaks depends also of these conditions. The high quality of the $c_{p}^{\prime \prime}$ curves is due to the high resolution of the nanocalorimeter. This bring the experimental basis for a thermodynamic and dynamic treatment of the glass transition process.

\section{Perspectives}

The prospects will concern the use of this sensitive nanocalorimetric instrumentation in order to deeply investigate the dynamic and thermodynamic aspects of the glass transition on model systems such as PVAc. In particular, we envisage three different paths for such investigation:

-firstly, a systematic study on the frequency dependence of the glass temperature $T_{g}$ will be done. This will be made on the available interval of thermal frequencies, i.e. between about $8 \mathrm{mHz}$ and $3 \mathrm{~Hz}$. These frequencies can be for a part comparable to that use in TMDSC and a systematic comparison between TMDSC and ac-calorimetry may be suitable. The activation energy which can be extracted from these data will be compared to that classically extracted from DSC measurements on the same systems.

-secondly, the dynamic of the glass transition will be investigated as a function of the different thermal histories on the system. At fixed frequency, the rate of temperature cooling, heating and different aging times at different constant temperature will be used to probe the dynamic of the glass transition not only on the change in $T_{g}$ but on the width of the glass transition interval. This width is connected to the half-width of the $c_{p}^{\prime \prime}$ peaks for example. This will bring information on the cooperativity of the structural recovery process, and theories such as Donth's approach will be tested $[20,21]$.

-thirdly, the stabilization process observed across a fine decrease of the heat capacity (modulus and phase) along time will be more deeply examined at different temperature. Connections will be established between this stabilization process, the evolution in time of the distribution of relaxation times and the resulting increase of cooperativity observable in the evolution of the $c_{p}^{\prime \prime}$ half-width.

\section{CONCLUSION}

We have developed a new highly sensitive acnanocalorimeter. The instrument is based on a microsensor made with the microfabrication technologies. The thermal and electronic conditioning of the sensor have been thoroughly described. The low noise and highly stable electronic detection chain based on pseudodifferential thermometric mode, as well as the low mass of the sensitive area of the sensor, lead to a very high resolution in the detection of the heat capacity variations of a sample. The good stability and repeatability of the apparatus are particularly adapted to the study of the glass transition. It could be more generally used in the study of slowly relaxing systems. This dynamic nanocalorimeter provides the amplitude and phase of the temperature oscillation of sample with its addenda. A thermal model of the device has been developed in order to extract the real and imaginary part of the frequency dependent specific heat of the sample over more than two decade of frequency range, which is not current for this type of accalorimeter. First measurements on the glass transition of PVAc glass former have been carried out showing the capability of the instrument to investigate the dynamic and thermodynamic aspect of the glass transition. 


\section{ACKNOWLEDGMENTS}

We would like to address a special thanks to Jacques Chaussy for having initiated this project, to Jean-Louis Bret who gave all the solutions for high stability and low noise analogical electronic chain, and to Pierre BrosseMaron who gave reliable solutions for the mechanical conditioning of the sensor. We also thank the Thermodynamics and Biophysics of Small Systems team for the host of the $\mathrm{PhD}$ and the scientific surroundings. The know-how of the Thermometric Sensors and Calorimetry group, the Electronics group, and the Cryogenics group of the laboratory have been plainly used across the work of the technicians and engineers that we thank a lot for their help. We would also like to gratefully thanks Professor G. McKenna who gave us a long time ago the PVAc samples. Finally we acknowledge the financial support of the SCAC (Embassy of France in Morocco: Service of Cooperation and Cultural Action), Ministry of Higher Education of Morocco, the Action intégrée CNRS-CNRST, the Région Rhone-Alpes, and the TPS team for their financial support during the $\mathrm{PhD}$ thesis.

[1] J. E. K. Schawe. Thermochim. Acta, 260:1-16, 1995.

[2] J.-L. Garden. Thermochim. Acta, 452:85-105, 2007.

[3] N. O. Birge and S. R. Nagel. Phys. Rev. Lett., 54:26742677,1985

[4] N. O. Birge. Phys. Rev. B, 34:1631-1642, 1986.

[5] T. Christensen. Journal de Physique, Colloque C8, supplment au n12, Tome 46:C8-635-C8-637, 1985.

[6] M. Beiner, J. Korus, H. Lockwenz, K. Schrter, and E. Donth. Macromolecules, 29:5183-5189, 1996.

[7] E. H. Bentefour, C. Glorieux, M. Chirtoc, and J. Thoen. J. Appl. Phys., 93:9610-9614, 2003.

[8] J.-L. Garden. Thermochim. Acta, 460:85-87, 2007.

[9] J.-L. Garden and J. Richard. Thermochim. Acta, 461:57$66,2007$.

[10] H. Gobrecht, K. Hamann, and G. Willers. J. Phys. E: Sci. Instrum., 4:21-23, 1971.

[11] P.S. Gill, S.R. Sauerbrunn, and M. Reading. J. Therm.
Anal., 40:931-939, 1993.

[12] J. E. K. Schawe. Thermochim. Acta, 261:183-194, 1995.

[13] A. Hensel, J. Dobbertin, J. E. K. Schawe, A. Boller, and C. Schick. J. Therm. Anal., 46:935-954, 1996.

[14] J. E. K. Schawe. J. Therm. Anal., 47:475-484, 1996.

[15] O. Bustin and M. Descamps. J. Chem. Phys., 110:1098210992, 1999.

[16] G. salvetti, C. Cardelli, C. Ferrari, and E. Tombari. Thermochim. Acta, 364:11-22, 2000.

[17] S. Weyer, M. Merzlyakov, and C. Schick. Thermochim. Acta, 377:85-96, 2001.

[18] L. Carpentier, O. Bustin, and M. Descamps. J. Phys. D: Appl. Phys., 35:402-408, 2002.

[19] R. Garkova, Ts. Vassilev, C. Rssel, and I. Avramov. J. Non-Cryst. Solids, 333:240-245, 2004.

[20] A. Saiter, L. Delbreilh, H. Couderc, K. Arabeche, A. Schnhals, and J.-M. Saiter. Phys. Rev.E, 81:0418051-041805-8, 2010.

[21] O. Gulbiten, J. C. Mauro, and P. Lucas. J. Chem. Phys., 138:244504-1-244504-7, 2013.

[22] P.F. Sullivan and G. Seidel. Phys. Rev., 173:679-685, 1968.

[23] J.-L. Garden, H. Guillou, A. F. Lopeandia, J. Richard, J.-S. Heron, G. M. Souche, F. R. Ong, B. Vianay, and O. Bourgeois. Thermochim. Acta, 492:16-28, 2009.

[24] S. Tagliati, V. M. Krasnov, and A. Rydh. Rev. Sci. Instrum., 83:055107-1-055107-11, 2012.

[25] I. Hatta and A. J. Ikushima. Jap. J. Appl. Phys., 20:1995-2011, 1981.

[26] E. Gmelin. Thermochim. Acta, 3281:1-27, 1997.

[27] Y. Kraftmakher. Phys. Rep., 356:1-117, 2002.

[28] Y.-H. Jeong. Thermochim. Acta, 304/305:67-98, 1997.

[29] M. Castro and J. A. Puertolas. Thermochim. Acta, 402:159-168, 2003

[30] H. Huth, A. A. Minakov, and C. Schick. J. Polym. Sci. B Polym. Phys., 44:2996-3005, 2006.

[31] D. Zhou, H. Huth, Y. Gao, G. Xue, and C. Schick. Macromolecules, 41:7662-7666, 2008.

[32] K. Xiao, J. M. Gregoire, P. J. McCluskey, and J. J. Vlassak. Rev. Sci. Instrum., 83:114901-1-114901-15, 2012.

[33] S. Tagliati and A. Rydh. Thermochim. Acta, 522:66-71, 2011.

[34] E. Tombari, S. Presto, G. Salvetti, and G. P. Johari. J. Chem. Phys, 117:8436-8441, 2002.

[35] M. Beiner, S. Reissig, K. Schrter, and E. Donth. Rheol. Acta, 36:187-196, 1997. 